\title{
Availability of right femoral vein as a route for tunneled hemodialysis catheterization
}

This article was published in the following Dove Press journal:

Medical Devices: Evidence and Research

\author{
Mitsutoshi Shindo' \\ Hiroaki Takemae ${ }^{2}$ \\ Takafumi Kubo² \\ Masatsugu Soeno ${ }^{2}$ \\ Tetsuo Ando 2 \\ Yoshiyuki Morishita' \\ 'Division of Nephrology, First \\ Department of Integrated Medicine, \\ Saitama Medical Center, Jichi \\ Medical University, Saitama, Japan; \\ ${ }^{2}$ Department of Dialysis and \\ Transplant Surgery, Hidaka Hospital, \\ Gunma, Japan
}

Purpose: This study was performed to investigate the primary patency rate and catheterrelated problems associated with use of the femoral vein as a route for tunneled hemodialysis catheterization compared with those of the right internal jugular vein as the first-choice route in patients undergoing maintenance hemodialysis.

Patients and methods: Twenty-two patients underwent placement of indwelling tunneled hemodialysis catheters in the right internal jugular vein as the first option for maintenance hemodialysis, and 20 patients underwent placement in the right femoral vein as the second option. The primary patency rate of the catheters and catheter-related problems at 1, 3, 6, and 12 months after placement were investigated.

Results: The 1-, 3-, 6-, and 12-month primary patency rates of the tunneled hemodialysis catheters in the right internal jugular vein were $95.5 \%, 95.5 \%, 81.3 \%$, and $58.3 \%$. The primary patency rates of the catheters in the right femoral vein were $95.0 \%, 89.5 \%, 86.7 \%$, and $66.7 \%$. There were no statistically significant differences in the primary patency rates at $1,3,6$, and 12 months or in catheter-related problems between the right internal jugular vein and right femoral vein.

Conclusion: The primary patency rate and catheter-related problems of indwelling tunneled hemodialysis catheters placed in the right femoral vein were not different from those in the right internal jugular vein in patients undergoing maintenance hemodialysis. These results suggest that the right femoral vein might be a useful option for placement of indwelling tunneled hemodialysis catheters in patients undergoing maintenance hemodialysis.

Keywords: tunneled hemodialysis catheter, right femoral vein, primary patency rate, catheterrelated problems

\section{Introduction}

The number of patients with end-stage chronic kidney disease requiring hemodialysis is increasing worldwide. ${ }^{1}$ Vascular access is necessary for conductance of hemodialysis. A native arteriovenous fistula or, when this is difficult to establish, an arteriovenous graft is considered the preferable vascular access for maintenance hemodialysis because these access sites are durable with repeated use and have less risk of access-related problems such as access failure and infection. However, many patients with end-stage chronic kidney disease have concomitant diseases that impair blood vessels, such as diabetes mellitus and arteriosclerosis. ${ }^{2}$ In these patients, a tunneled hemodialysis catheter may be selected for vascular access because an arteriovenous fistula or arteriovenous graft would be difficult to establish using the patient's impaired blood vessels. In addition, a tunneled hemodialysis catheter is reportedly useful for vascular access in patients with
Correspondence: Yoshiyuki Morishita Division of Nephrology, First Department of Integrated Medicine, Saitama Medical Center, Jichi Medical University, I-847 Amanuma, Omiya-ku, Saitama 330-8503, Japan

Tel +81486472 III

Fax +8I 486476831

Email ymori@jichi.ac.jp 
acute kidney injury, ${ }^{3}$ patients with end-stage chronic kidney disease who require temporary vascular access for transient hemodialysis before renal transplantation, and patients who must wait for the development of an arteriovenous fistula or arteriovenous graft for maintenance hemodialysis after an operation. A previous study showed that a tunneled hemodialysis catheter should be employed in patients who are expected to have an indwelling hemodialysis catheter for more than 14 days. $^{4}$

The right internal jugular vein has been recommended as the first option for placement of a tunneled hemodialysis catheter because it runs in a straight line at a superficial site in the neck, resulting in high accessibility and fewer catheter-related problems. ${ }^{5}$ For patients with problems such as occlusion of the right internal jugular vein, insertion of a pacemaker in the right chest, or difficulty maintaining the required surgical position, use of the left internal jugular vein or bilateral femoral veins may be considered..$^{5}$ Among these veins, the left internal vein and left femoral vein run crookedly; thus, they may be associated with a risk of catheter-related complications. Indeed, the left internal jugular vein reportedly has a higher risk of catheter-related difficulties than the right internal jugular vein. ${ }^{5-7}$ Although several studies have been performed to investigate the femoral vein as a route for tunneled hemodialysis catheterization in patients undergoing maintenance hemodialysis, the availability and risk of the right femoral vein for an indwelling tunneled hemodialysis catheter has not been fully clarified. ${ }^{8-11}$ In the present study, we retrospectively compared the primary patency rate and catheter-related problems of the right femoral vein versus the right internal jugular vein as the first-option route for tunneled hemodialysis catheterization in patients undergoing maintenance hemodialysis.

\section{Materials and methods}

This retrospective cohort study was performed in a single dialysis center to investigate the availability of the right femoral vein as a route for tunneled hemodialysis catheterization in patients undergoing maintenance hemodialysis from May 2013 to June 2016 in Hidaka Hospital. This study was performed in accordance with the ethical principles of the Declaration of Helsinki and was approved by the ethics committee of Hidaka Hospital. The patient consent to review their medical records was not required by the ethics committee of Hidaka Hospital because this study examined a retrospective cohort by analyzing the past medical history. The patient data were kept confidential.

\section{Tunneled hemodialysis catheter}

The tunneled hemodialysis catheter used for both the bilateral internal jugular veins and bilateral femoral veins was $19 \mathrm{~cm}$ long with a 12.5-French bore size (Soft-Cell; C. R. Bard Inc., Murray Hill, NJ, USA) (Figure 1). All catheter insertions and placements were performed by transplant surgeons from our

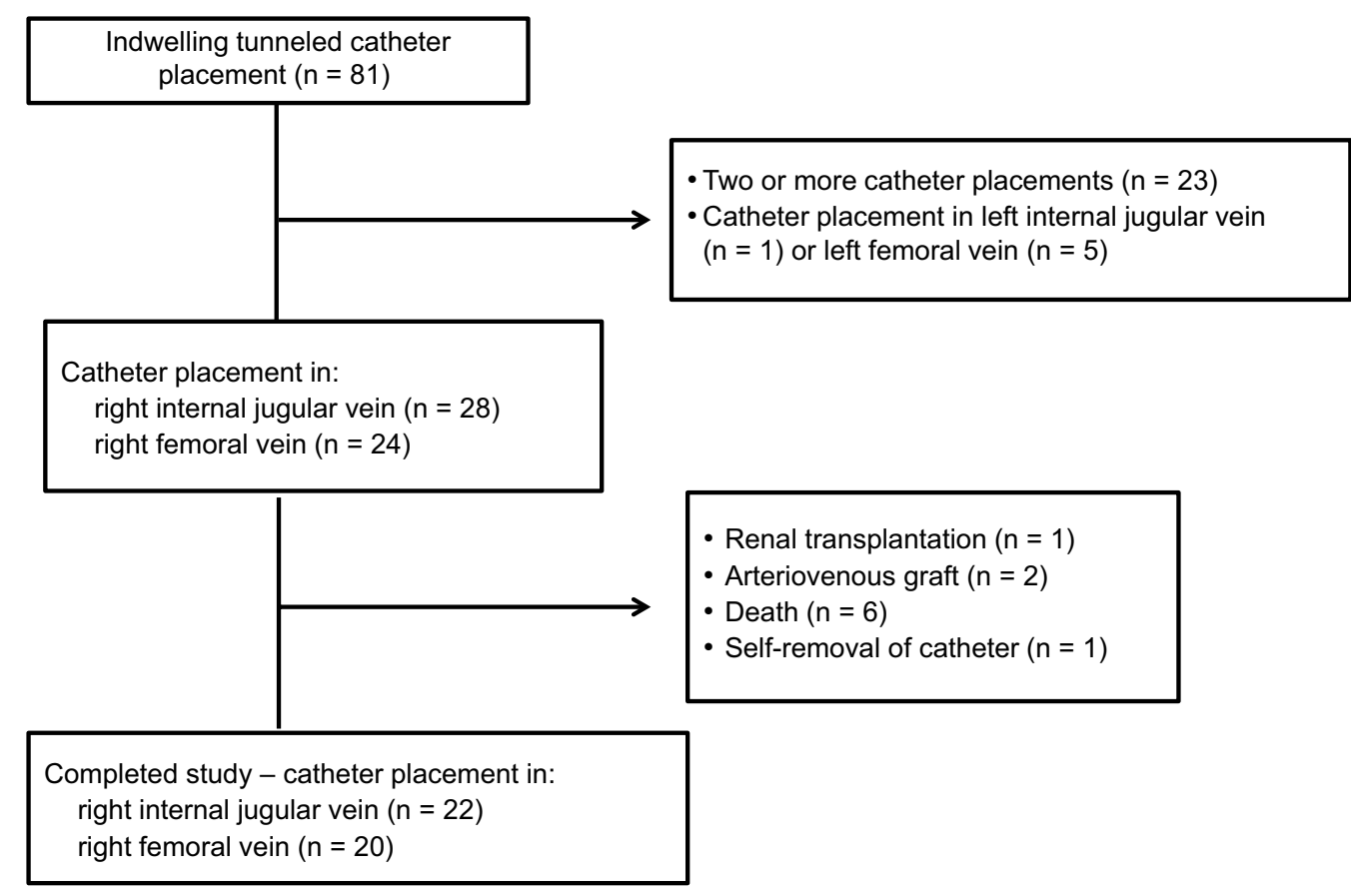

Figure I Patient flow chart. 
hemodialysis center using a Seldinger guidewire technique with ultrasound guidance in the fluoroscopy suite of Hidaka Hospital. In accordance with the Japanese Society for Dialysis Therapy Guidelines of Vascular Access Construction and Repair for Chronic Hemodialysis, the right internal jugular vein was selected as the first-choice access vein because it has high accessibility and a straight route in the neck, resulting in high patency and fewer catheter-related problems such as catheter dysfunction and infection. ${ }^{5}$ In patients with complications such as occlusion of the right internal jugular vein, insertion of a pacemaker in the right chest, or difficulty maintaining the required surgical position for placement of an indwelling tunneled hemodialysis catheter, the right femoral vein was selected as the second option. The tip of the indwelling tunneled hemodialysis catheter in the right femoral vein was confirmed to be localized in the inferior vena cava, on the more cranial side of the joint portion of the right and left femoral veins. The exit site of the indwelling tunneled hemodialysis catheter in the right femoral vein was located at the skin $8-10 \mathrm{~cm}$ caudal to the right inguinal ligament. When use of the right internal jugular vein and right femoral vein was problematic, the left internal jugular vein and left femoral vein were selected as the third and fourth options, respectively. The tunneled hemodialysis catheter was used during hemodialysis and locked with 2,000 units $(2.0 \mathrm{ml})$ of heparin solution in both the venous- and arterial-side tubes after each hemodialysis session. The patients were advised to maintain a clean vascular access site and avoid bending the tunneled hemodialysis catheter. The patients could bend their hips, but they were advised not to squat.

\section{Primary patency rate of tunneled hemodialysis catheter and catheter- related problems}

The primary patency rate of the tunneled hemodialysis catheters and catheter-related problems, including catheter dysfunction and infection, were investigated at 1, 3, 6, and 12 months after catheter insertion. Catheter dysfunction was defined when the blood flow rate was $<120 \mathrm{ml} / \mathrm{min}$ despite an attempt to salvage the catheter by urokinase injection into the catheter. When catheter dysfunction was diagnosed, the catheters were removed. Catheter-related infections included exit site infections and bloodstream infections. An exit site infection was defined as the presence of flare, swelling, and existence of pus at the exit site in the skin. When an exit site infection was diagnosed, the exit site was washed with normal saline solution. A bloodstream infection was diagnosed when a blood culture was positive for bacterial pathogens and other infectious sites were not observed or not likely to be the origin of the bacteria. When a bloodstream infection was diagnosed, the catheters were removed.

\section{Patients}

The patient flow chart is shown in Figure 1. Eighty-one patients underwent maintenance hemodialysis with an indwelling tunneled hemodialysis catheter in the internal jugular vein or femoral vein from May 2013 to June 2016 in Hidaka Hospital. Of these 81 patients, 23 were excluded from this study because they had a history of indwelling tunneled hemodialysis catheter placement before the study period, and 6 were excluded because the tunneled hemodialysis catheter was placed in the left internal jugular vein as a third option (1 patient) or left femoral vein as a fourth option ( 5 patients). During the observation period, we also excluded 10 patients who underwent renal transplantation (1 patient), underwent arteriovenous grafting ( 2 patients), died of catheter-unrelated diseases (6 patients), and removed the tunneled hemodialysis catheter because of delirium (1 patient). The remaining 42 patients who underwent placement of an indwelling tunneled hemodialysis catheter in the right internal jugular vein as the first option (22 patients) and right femoral vein as the second option (20 patients) were analyzed (Figure 2). Among these 42 patients, 23 patients were followed up for 12 months, 8 patients were followed up for 6 months, 10 patients were followed up for 3 months, and 1 patient was followed up for 1 month (Figure 2).

\section{Parameters of hemodialysis}

Single pooled KT/V (spKT/V) values were calculated each month by previously reported formulae. ${ }^{12}$ The median spKT/V was calculated for each patient after placement of the indwelling tunneled hemodialysis catheter.

\section{Statistical analysis}

Data are expressed as median and range. Statistical analysis was performed using the Mann-Whitney $\mathrm{U}$ test; a $p$ value of $<0.05$ was considered statistically significant. Logistic regression analysis was used to detect independent predictors of catheter survival.

\section{Results}

The 42 analyzed patients underwent placement of indwelling tunneled hemodialysis catheters because of problems associated with their existing arteriovenous fistula or arteriovenous graft, including occlusion (18 patients), steal syndrome (3 patients), rupture or varicose of the arteriovenous fistula 


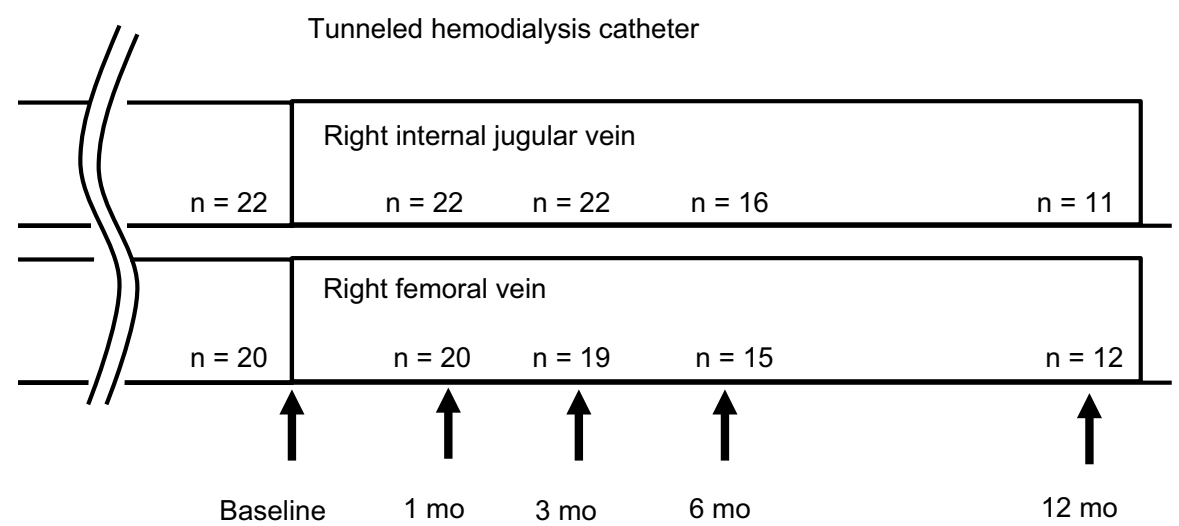

Primary patency rate, catheter-related problems

Figure 2 Diagram of study design.

Abbreviation: mo, month.

(4 patients), severe puncture pain ( 2 patients), and difficult general conditions for creation of the arteriovenous fistula or arteriovenous graft such as a bed-bound status (6 patients), marked edema ( 5 patients), and mental disorders (4 patients). Among the 42 patients, 22 underwent placement of the indwelling tunneled hemodialysis catheter in the right internal jugular vein as the first option. Among these 22 patients, 18 $(81.8 \%)$ could ambulate and $4(18.2 \%)$ were bedridden or had very low activity. The remaining 20 patients underwent placement in the right femoral vein as the second option because of occlusion of the right internal jugular vein ( 5 patients), presence of a pacemaker (1 patient) or central vein port (1 patient) in the right chest, or difficulty maintaining the required surgical position for the following reasons: bedridden status (6 patients), mental disorders (4 patients), and neck diseases such as cervical vertebral syndrome (3 patients). Among these 20 patients, $12(60.0 \%)$ could ambulate and 8 $(40.0 \%)$ were bedridden or had very low activity. The details of the patients and their hemodialysis conditions are summarized in Table 1. The patients' mean body mass index was $20.3 \pm 6.2 \mathrm{~kg} / \mathrm{m}^{2}$. No major complications occurred during catheter insertion, including hematoma, air embolism, or pneumothorax formation, in all patients in both the right internal jugular vein and right femoral vein groups.

\section{Hemodialysis conditions}

The blood flow rate from the tunneled hemodialysis catheter was set at $150 \mathrm{ml} / \mathrm{min}$ in all patients to prevent interruption of the hemodialysis session due to catheter dysfunction. The $\mathrm{KT} / \mathrm{V}$ of patients who underwent placement of indwelling tunneled hemodialysis catheters in the right internal jugular vein was 1.40 (1.2-1.62). The KT/V of patients who underwent placement of indwelling tunneled hemodialysis catheters in the right femoral vein was 1.19 (0.91-1.47).

\section{Primary patency rate of tunneled hemodialysis catheter}

The primary patency rate of the catheters in the right internal jugular vein was $95.5 \%(21 / 22)$ at 1 month, 95.5\% (21/22) at 3 months, $81.3 \%(13 / 16)$ at 6 months, and $58.3 \%(7 / 11)$ at 12 months. The primary patency rate of the catheters in the right femoral vein was $95.0 \%(19 / 20)$ at 1 month, $89.5 \%$ $(17 / 19)$ at 3 months, $86.7 \%(13 / 15)$ at 6 months, and $66.7 \%$ $(8 / 12)$ at 12 months. There was no statistically significant difference in the patency of the tunneled hemodialysis catheters in the right internal jugular vein versus right femoral vein at 1, 3, 6, and 12 months (Table 2). No factors, including age, sex, body mass index, dialysis condition, spKT/V, coexisting diseases, and medications, were significantly associated with catheter survival in patients with an indwelling tunneled hemodialysis catheter in either the right internal jugular vein or right femoral vein.

\section{Catheter-related problems}

Dysfunction of the tunneled hemodialysis catheter was observed in 3 patients with an indwelling tunneled hemodialysis catheter in the right internal jugular vein and 3 patients with a catheter in the right femoral jugular vein (Figure 3). The catheters were removed from these 6 patients. Two patients with an indwelling tunneled catheter in the right 
Table I Patients' characteristics

\begin{tabular}{|c|c|c|c|}
\hline & $\begin{array}{l}\text { Right internal jugular } \\
\text { vein }(n=22)\end{array}$ & $\begin{array}{l}\text { Right femoral vein } \\
(n=20)\end{array}$ & $p$ value \\
\hline Sex (male/female) & $9 / 13$ & $7 / 13$ & 0.39 \\
\hline Age (years) & $74.5(29.2-90.2)$ & $72.5(49.3-88.1)$ & 0.42 \\
\hline Body weight (kg) & $49.6(30.6-85.0)$ & $50.0(36.0-69.3)$ & 0.47 \\
\hline Body mass index $\left(\mathrm{kg} / \mathrm{m}^{2}\right)$ & $21.5(14.4-29.1)$ & $22.8(15.7-29.6)$ & 0.04 \\
\hline Dialysis duration (years) & $\mathrm{I} .8(0.1-35.4)$ & $4.6(0.2-33.4)$ & 0.19 \\
\hline Dialysis sessions (times/week) & $3.0(2.0-3.0)$ & $3.0(2.0-3.0)$ & 0.30 \\
\hline Dialysis time (hours/session) & $4.0(3.0-4.0)$ & $4.0(3.0-4.0)$ & 0.42 \\
\hline Blood flow rate $(\mathrm{ml} / \mathrm{min})$ & 150 & 150 & \\
\hline spKT/V & $1.40(1.2-1.62)$ & $1.19(0.91-1.47)$ & 0.52 \\
\hline \multicolumn{4}{|l|}{ Initial nephropathy } \\
\hline Diabetes & 14 & 6 & 0.03 \\
\hline Chronic glomerulonephritis & 3 & 7 & 0.11 \\
\hline Nephrosclerosis & I & I & 0.97 \\
\hline Nephrolithiasis & I & 0 & 0.17 \\
\hline Ureter tumor & 0 & I & 0.32 \\
\hline Renal cell carcinoma & 0 & I & 0.32 \\
\hline Renal sclerosis & I & 0 & 0.17 \\
\hline Polycystic kidney & 0 & 1 & 0.32 \\
\hline Unknown & 2 & 3 & 0.57 \\
\hline \multicolumn{4}{|l|}{ Coexisting disease } \\
\hline Diabetes & 20 & 11 & 0.01 \\
\hline Hypertension & 23 & 19 & 0.12 \\
\hline Cardiovascular disease* & 15 & 7 & 0.02 \\
\hline \multicolumn{4}{|l|}{ Medications } \\
\hline Aspirin (only) & 4 & 2 & 0.20 \\
\hline Ticlopidine (only) & 3 & I & 0.16 \\
\hline Anticoagulant (only) & I & 4 & 0.08 \\
\hline DAPT & 6 & 2 & 0.07 \\
\hline DAPT + anticoagulant & I & 0 & 0.17 \\
\hline Ticlopidine + anticoagulant & 0 & 2 & 0.08 \\
\hline Hemoglobin $(g / d L)$ & $9.8(7.5-12.4)$ & $9.6(7.4-11.8)$ & 0.06 \\
\hline Serum albumin $(g / d L)$ & $3.1(1.3-3.9)$ & $2.7(1.7-3.8)$ & 0.32 \\
\hline
\end{tabular}

Note: Data are presented as $\mathrm{n}$ or median (range). ${ }^{*}$ Cardiovascular disease includes ischemic heart disease, heart valve disease, and arrhythmia.

Abbreviations: DAPT, dual-antiplatelet therapy; spKT/V, single pooled KT/V.

Table 2 Primary patency rate of tunneled hemodialysis catheter

\begin{tabular}{llll}
\hline & $\begin{array}{l}\text { Right internal } \\
\text { jugular vein }\end{array}$ & $\begin{array}{l}\text { Right femoral } \\
\text { vein }\end{array}$ & p-value \\
\hline I month & $21 / 22(95.5 \%)$ & $19 / 20(95.0 \%)$ & 0.48 \\
3 months & $21 / 22(95.5 \%)$ & $17 / 19(89.5 \%)$ & 0.31 \\
6 months & $13 / 16(81.3 \%)$ & $13 / 15(86.7 \%)$ & 0.35 \\
I2 months & $7 / 11(58.3 \%)$ & $8 / 12(66.7 \%)$ & 0.46 \\
\hline
\end{tabular}

internal jugular vein (1 patient) and right femoral vein (1 patient) underwent catheter removal due to catheter-related bloodstream infection (Figure 4). No exit site infections were observed in patients with an indwelling tunneled hemodialysis catheter in either the right internal jugular vein or right femoral vein. There was no significant difference in catheter-related problems such as catheter-dysfunction and catheter-related infection between the right internal jugular vein and right femoral vein groups (Table 3).

\section{Discussion}

In this study, there was no significant difference in the primary patency rate at $1,3,6$, and 12 months or in catheterrelated problems such as catheter dysfunction and infection between the tunneled hemodialysis catheters in the right internal jugular vein versus right femoral vein among patients undergoing maintenance hemodialysis. These results suggest that the right femoral vein might be a useful option for placement of indwelling tunneled hemodialysis catheters in patients undergoing maintenance hemodialysis.

The right internal jugular vein is reportedly the most effective first option for indwelling tunneled hemodialysis catheters because of its higher rate of primary patency. ${ }^{89} \mathrm{~A}$ 


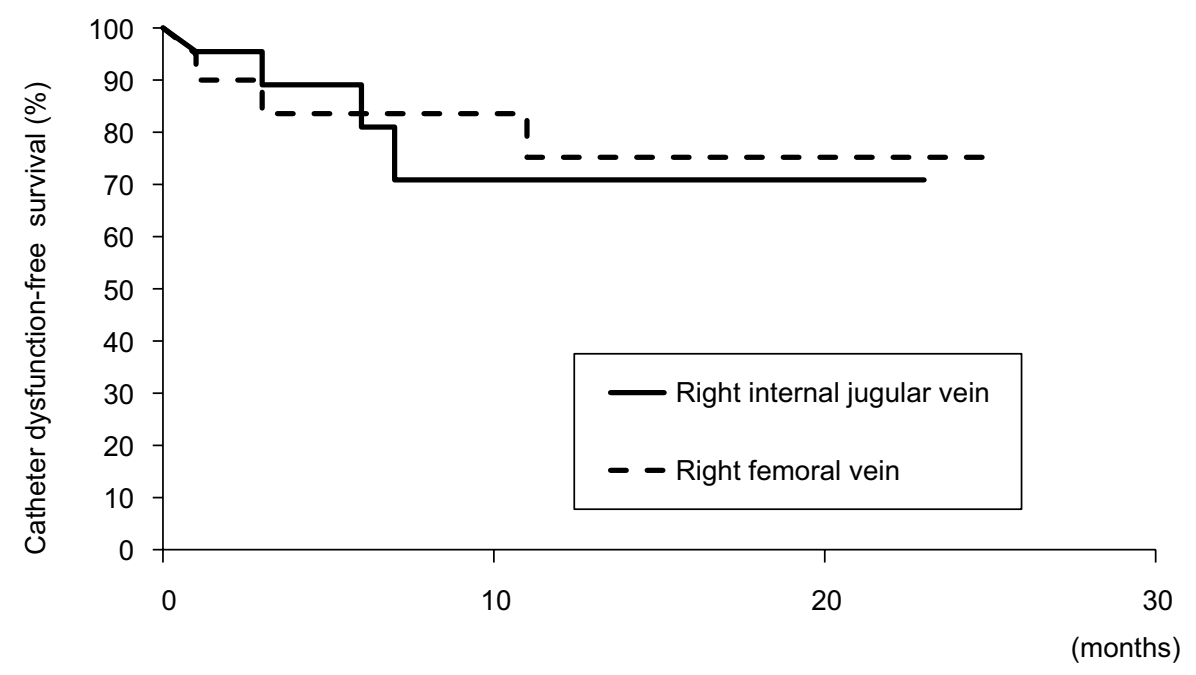

Figure 3 Kaplan-Meier curves for catheter dysfunction.

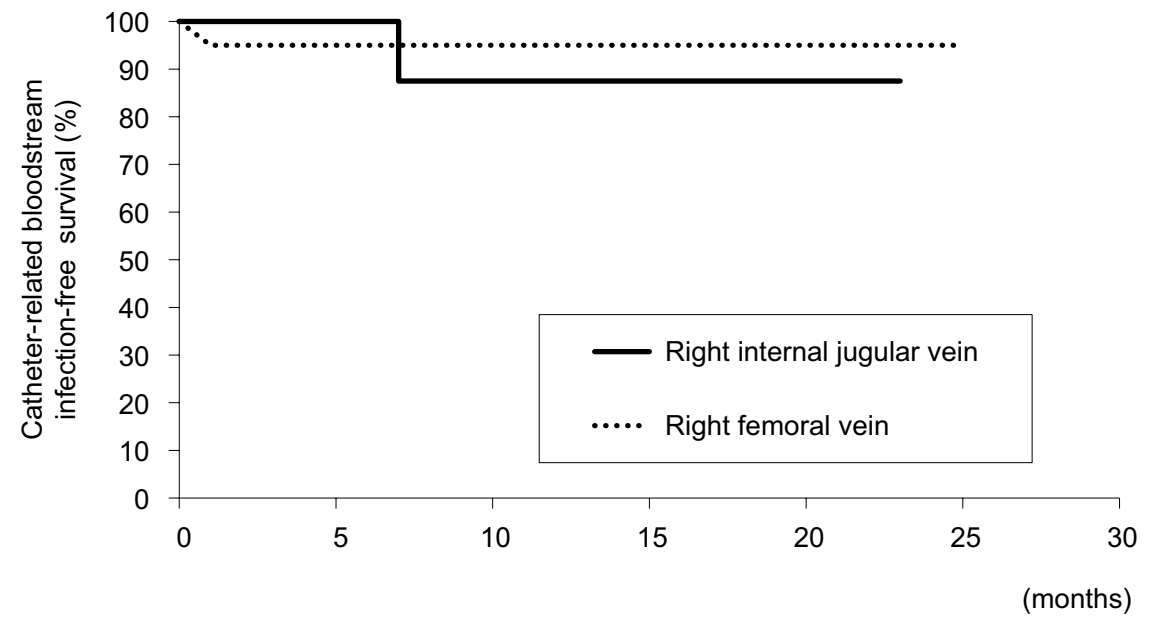

Figure 4 Kaplan-Meier curves for catheter-related bloodstream infections.

Table 3 Catheter-related problems

\begin{tabular}{llll}
\hline & $\begin{array}{l}\text { Right } \\
\text { internal } \\
\text { jugular vein }\end{array}$ & $\begin{array}{l}\text { Right } \\
\text { femoral } \\
\text { vein }\end{array}$ & p-value \\
\hline $\begin{array}{l}\text { Dysfunction } \\
\text { Catheter-related infections }\end{array}$ & $3 / 22(13.6 \%)$ & $3 / 20(15.0 \%)$ & 0.46 \\
$\begin{array}{l}\text { Bloodstream infection } \\
\text { Exit site infection }\end{array}$ & $1 / 22(4.5 \%)$ & $1 / 20(5.0 \%)$ & 0.49 \\
\hline
\end{tabular}

Note: "-" indicates not calculated.

previous study showed that the catheter patency rate at 180 days after indwelling catheter placement was $67.0 \%$ in the right internal jugular vein and $14.0 \%$ to $27.1 \%$ in the femoral vein. ${ }^{8}$ In contrast, the catheter-related infection rate was not significantly different between the right internal jugular vein and right femoral vein. ${ }^{8-11}$ In the present study, the 6-month and 12-month primary patency rates of tunneled hemodialysis catheters in the femoral vein were high at $86.7 \%$ and $66.7 \%$, respectively, compared with those of previous study. ${ }^{8}$ The primary patency rate and catheter-related infection rate were not different between the right internal jugular vein and right femoral vein. One study revealed that the hemodialysis catheter-related infection rate was higher in the femoral vein than right internal jugular vein in patients with obesity (body mass index of $>28 \mathrm{~kg} / \mathrm{m}^{2}$ ). ${ }^{11}$ In the present study, the low to moderate body mass index of the studied patients may have contributed to the improved primary patency rate and catheterrelated problems, including catheter dysfunction and infection, in the right femoral vein. In addition, we employed a $19-\mathrm{cm}-$ long tunneled hemodialysis catheter for both the right femoral vein and right internal jugular vein because it was long enough for placement in the right femoral vein of all studied patients without obesity. A longer tunneled hemodialysis catheter $(35 \mathrm{~cm})$ reportedly causes more catheter-related problems than does a shorter catheter. ${ }^{13}$ The short length of the tunneled hemo- 
dialysis catheter in this study may have also contributed to the improved primary patency rate and catheter-related problems in the right femoral vein. Another possibility is that the advice regarding catheter care provided by the medical staff and the performance of catheter care by the patients in this study might have also contributed to the improved catheter condition. One study revealed that catheter care and antimicrobial treatment performed by the dialysis center staff and patients may contribute to an improved catheter prognosis. ${ }^{9}$ In the present study, no major complications occurred including hematoma, air embolism, or pneumothorax formation in all patients regardless of catheter location during either the catheter insertion or removal process, and bleeding around catheters during the observation period. These results suggest that the right femoral vein might be a feasible route for placement of an indwelling tunneled hemodialysis catheter and may not have a higher rate of catheter-related problems. The spKT/V was low in patients who underwent placement of indwelling tunneled hemodialysis catheters in the right internal jugular vein (1.40 [1.20-1.62]) and in the right femoral vein (1.19 [0.91-1.47]). An increased blood flow rate or prolonged dialysis time may be necessary for adequate long-term dialysis.

\section{Limitations}

This study had some limitations. First, it was a single-center retrospective study, and the number of patients was small. Second, the rate of blood flow from the tunneled hemodialysis catheter was set at $150 \mathrm{ml} / \mathrm{min}$ in all patients to prevent interruption of the hemodialysis session due to catheter dysfunction, which may have resulted in low dialysis efficiency. Large-scale, multicenter trials are required to investigate the availability and safety of the right femoral vein for placement of an indwelling tunneled hemodialysis catheter with an increased blood flow rate.

\section{Conclusion}

The primary patency rate and catheter-related problems of tunneled hemodialysis catheters in the right femoral vein were not different from those in the right internal jugular vein as a first option in patients undergoing maintenance hemodialysis. These results suggest that the right femoral vein might be a useful option for indwelling tunneled hemodialysis catheters in patients undergoing maintenance hemodialysis.

\section{Acknowledgments}

The authors thank the members of the Division of Nephrology, First Department of Integrated Medicine, Saitama Medical Center, Jichi Medical University, and Department of Dialysis and Transplant Surgery, Hidaka Hospital, Gunma, Japan. The authors also thank Angela Morben, DVM, ELS, from Edanz Group (www.edanzediting.com/ac), for editing a draft of this manuscript.

This study was supported by the Division of Nephrology, First Department of Integrated Medicine, Saitama Medical Center, Jichi Medical University, Saitama, Japan.

\section{Author contributions}

All authors contributed toward data analysis, drafting and revising the paper and agree to be accountable for all aspects of the work.

\section{Disclosure}

The authors report no conflicts of interest in this work.

\section{References}

1. Liyanage $T$, Ninomiya $T$, Jha $\mathrm{V}$, et al. Worldwide access to treatment for end-stage kidney disease: a systematic review. Lancet. 2015;385(9981): 1975-1982.

2. US Renal Data System. USRDS 2009 Atlas of Chronic Kidney Disease and End-Stage Renal Disease in the United States, Volume 2, Chapter 2: Incidence \& prevalence of ESRD. Bethesda, MD: National Institutes of Health, National Institute of Diabetes and Digestive and Kidney Diseases; 2009.

3. Coryell L, Lott JP, Stavropoulos SW, et al. The case for primary placement of tunneled hemodialysis catheters in acute kidney injury. $J$ Vasc Interv Radiol. 2009;20(12):1578-1581.

4. Weijmer MC, Vervloet MG, ter Wee PM. Compared to tunnelled cuffed haemodialysis catheters, temporary untunnelled catheters are associated with more complications already within 2 weeks of use. Nephrol Dial Transplant. 2004;19(3):670-677.

5. Kukita K, Ohira S, Amano I, et al. 2011 update Japanese Society for Dialysis Therapy guidelines of vascular access construction and repair for chronic hemodialysis. Ther Apher Dial. 2015;19(Suppl 1):1-39.

6. Khwaja A. KDIGO clinical practice guidelines for acute kidney injury. Nephron Clin Pract. 2012;120(4):c179-c184.

7. Engstrom BI, Horvath JJ, Stewart JK, et al. Tunneled internal jugular hemodialysis catheters: impact of laterality and tip position on catheter dysfunction and infection rates. J Vasc Interv Radiol. 2013;24(9): 1295-1302.

8. Maya ID, Allon M. Outcomes of tunneled femoral hemodialysis catheters: comparison with internal jugular vein catheters. Kidney Int 2005;68(6):2886-2889.

9. Burton KR, Guo LL, Tan KT, et al. Patency of femoral tunneled hemodialysis catheters and factors predictive of patency failure. Cardiovasc Intervent Radiol. 2012;35(6):1396-1402.

10. Dugué AE, Levesque SP, Fischer MO, et al. Vascular access sites for acute renal replacement in intensive care units. Clin J Am Soc Nephrol. 2012;7(1):70-77.

11. Parienti JJ, Thirion M, Mégarbane B, et al. Femoral vs jugular venous catheterization and risk of nosocomial events in adults requiring acute renal replacement therapy: a randomized controlled trial. JAMA. 2008;299(20):2413-2422.

12. Shinzato T, Nakai S, Fujita Y, et al. Determination of $\mathrm{Kt} / \mathrm{V}$ and protein catabolic rate using pre- and postdialysis blood urea nitrogen concentrations. Nephron 1994;67(3):280-290.

13. Clark E, Kappel J, MacRae J, et al. Practical aspects of nontunneled and tunneled hemodialysis catheters. Can J Kidney Health Dis. 2016;3:2054358116669128 


\section{Publish your work in this journal}

Medical Devices: Evidence and Research is an international, peerreviewed, open access journal that focuses on the evidence, technology, research, and expert opinion supporting the use and application of medical devices in the diagnosis, monitoring, treatment and management of clinical conditions and physiological processes. The identification of novel devices and optimal use of existing devices which will lead to improved clinical outcomes and more effective patient management and safety is a key feature. The manuscript management system is completely online and includes a quick and fair peer-review system. Visit http://www. dovepress.com/testimonials.php to read real quotes from authors.

Submit your manuscript here: https://www.dovepress.com/medical-devices-evidence-and-research-journal 